\title{
Submitted from BMJ Quality Improving bowel preparation for
colonoscopy in a cost effective manner
}

\author{
Syed Anjum Gardezi, Clare Tibbatts
}

To cite: Gardezi SA, Tibbatts C. Improving bowel preparation for colonoscopy in a cost effective manner. BMJ Quality Improvement Reports 2017;6:u204560. w5376. doi:10.1136/ bmjquality.u204560.w5376

Received 16 June 2016 Revised 10 March 2017

\section{CrossMark}

Cardiff \& Vale university health board, Wales, United Kingdom

Correspondence to Syed Anjum Gardezi sanjliajk@gmail.com

\section{ABSTRACT}

Colonoscopy is a key investigation used to exclude large bowel pathologies including surveillance for CRC (Colorectal cancer) Poor bowel preparation (bowel prep) is one of the most important factors affecting its diagnostic yield. Different formulations of bowel prep are currently in use depending upon patient tolerance, indication \& co-morbidities. In University Hospital Llandough we retrospectively reviewed the outcome of colonoscopies performed over period of 3 months, in relation to the type and outcome of bowel preparations used. We implemented a change of patient instruction and pre-assessment of bowel preparation prescribed. We repeated the same measurements over 3 different cycles on 3 different occasions and compared the outcome.

We noticed that quality of bowel preparation noticeably improved from $80 \%$ to almost $93 \%$ if patients were given appropriate advice in a written format, prior to procedure. In addition to improvement in the quality of assessment \& reducing the number of repeat procedures, by changing the bowel preparation product and postage methods we estimated savings of almost $£ 150,000$ for the trust in a year.

\section{PROBLEM}

For optimal assessment of large bowel with colonoscopy, it is imperative to have a good bowel preparation with minimal faecal contamination. ${ }^{1}$ Poor bowel preparation is the leading cause of failed colonoscopy examinations. ${ }^{2}$ Numerous bowel cleansing agents are in use for this purpose; however there is a noticeable limitation to their effectiveness due to variations in their tolerability \& side effect profile. An inadequately prepared bowel can lead to missed diagnosis or need for repeat procedures \& investigations ${ }^{3} 4$ with a significant financial impact. ${ }^{5}$ A more standardised approach to the selection and use of these agents can be helpful in achieving optimal bowel cleansing \& assessment. University Hospital Llandough(UHL) is a district general hospital in Cardiff, Wales. UHL Gastroenterology department provides the majority of the outpatient endoscopy services including bowel screening for Cardiff and Vale University Health Board. While working in the gastroenterology department we looked at the outcome of colonoscopies performed over period of 3 months in 625 patients. We aimed to improve the quality of bowel preparation, and hence quality of the procedure, alongside provision of a cost effective service.

\section{BACKGROUND}

Before endoscopic procedures and radiological imaging of the bowel, oral bowel cleansing preparations have long been in use to minimise bowel contamination for optimal assessment.Inadequate bowel cleansing can lead to missed or delayed diagnosis, longer more difficult procedures \& need for repeat procedures. This in turn can have significant implications on the cost effectiveness of these procedures. The Joint advisory group on GI endoscopy(JAG) recommends a standard of excellent or adequate bowel prep in more than $90 \%$ cases to maximise pathology detection, minimise the need for additional procedures as per BCSP guidance. ${ }^{6}$

A number of different bowel preparation agents are currently being used in UK. These include:

Citrafleet (sodium picosulphate and magnesium citrate), Citramag (magnesium carbonateand citric acid),Klean Prep (polyethylene glycol or PEG),Moviprep (polyethylene glycol or PEG),Picolax (sodium picosulphate and magnesium citrate) ${ }^{7}$

An ideal bowel cleansing agent should not only be effective in cleansing the bowel, but also well tolerated and convenient to use, with minimal side effect profile. So far, there is no single ideal agent for all situations and therefore has to be chosen on an individual basis, depending upon co-morbidities, tolerability and nature of the procedure. ${ }^{8}$ Optimal results can be achieved if bowel preps are chosen and administered in an appropriate more standardised manner.

Polyethylene glycols (PEGs) are nonabsorbable solutions that pass through the bowel without absorption. They need to be 
taken in large amount of water and absorption of this can lead to fluid overload in predisposed individuals (e. g. those with Chronic Kidney Disease or Congestive Cardiac Failure). ${ }^{9}$

Oral phosphate based preparations are mainly effective by drawing large amount of water into the gut and don't need to be taken with larger volumes of fluid. They can however be associated with electrolyte imbalance and rapid fluid shifts, but are also cost effective. $^{1011}$

A good bowel prep gives adequate cleansing to the colon for optimal assessment. Different scales are used for scoring bowel preparation among different trusts with small variations and are based on the quality of bowel cleansing encountered during withdrawal of the scope. One of the most commonly used scale is the Boston Bowel Preparation Scale. ${ }^{12}$ In our trust we described bowel preparation on a scale of 1-5 where type $1 \& 2$ were considered adequate for examination while type 3-5 were considered suboptimal.

*Types of bowel prep: Clear Liquids/Type 1, Cloudy Liquids/Type 2, Liquids \& Semi Solids/Type3, Semi Solids and Solids /Type 4, Solids/Type 5

\section{BASELINE MEASUREMENT}

We reviewed the records of 625 colonoscopies over a period of 3 months looking particularly at the type of prep used, quality of bowel cleansing and if any repeat investigation was required due to poor cleansing.

$455(75 \%)$ of the patients had Kleen prep while 150 (25\%) had Moviprep, ie 100\% had PEG preparation. Quality of bowel cleansing was defined as Clear liquids, Cloudy liquids, Liquids/semi-solids, Semi-Solids/Solids and Solids \& labelled as Type 1-5 respectively where Type 1 \& Type 2 were considered as adequate. Results of the first audit showed that $490(80 \%)$ patients had adequate preparation (Type1, Type2) while 118(19\%) patients had inadequate preparation (Type3-5). 14 $(2.24 \%)$ patients needed repeat investigations either as a colonoscopy or CT and 6 of them were brought in to the hospital as an inpatient for bowel preparation on the next occasion (Figure 1).

\section{DESIGN}

We focused on improving the quality of bowel preparation as an indirect measure of optimal screening procedure. Secondary objective was to amend the type of preparation used and postage methods for outpatients in a cost effective manner without compromising the quality of procedure.

With my consultant/mentor, my fellow registrar in department and endoscopy booking office, we designed patient information leaflets about the appropriate use of bowel prep for optimal efficacy. We suggested a list of allowed foods, drinks and medications preceding the procedure. Along with that, we also designed an algorithm to help choose the most appropriate bowel
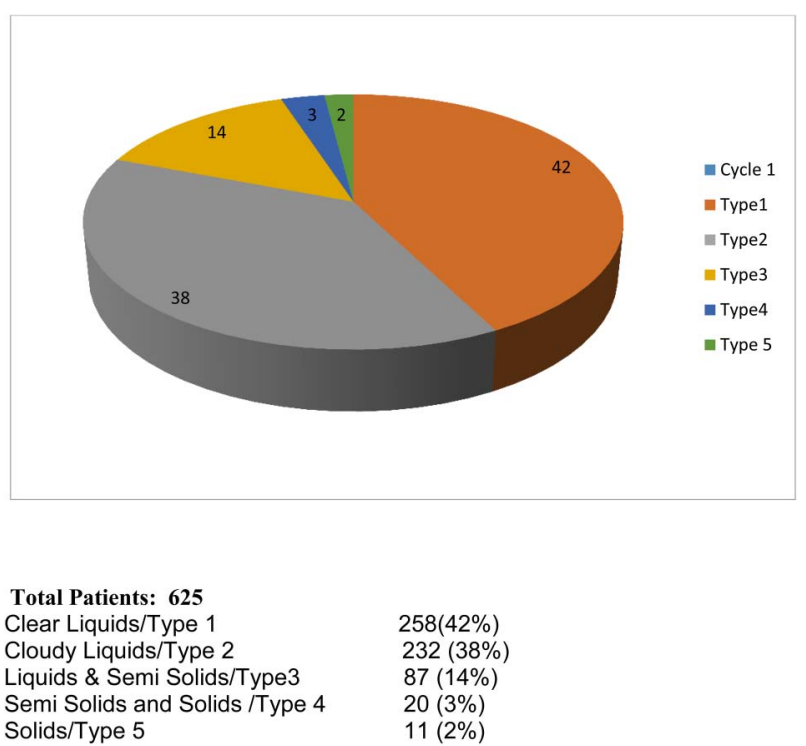

Figure 1 (Baseline measurements of bowel prep quality)

preparation, according to individual patient needs and cost effectiveness, moving away from using the more expensive PEG based preparations in all cases.

In terms of financial implications, we also reviewed the method of collecting or delivering the bowel preparation. A simple switch to increasing the numbers of direct bookings and posting the preparation sachets in a padded envelope, as opposed to a cardboard box, was extremely cost effective, and still legally acceptable in terms of medication delivery and prescription.

\section{STRATEGY}

\section{PDSA 1}

During the procedure it had been brought to attention many times that patient took the prescribed prep either inadequately or in a sub optimal manner. Also their diet in the preceding couple of days before the procedure influenced the quality of their bowel cleansing. In the first phase of our project we focused on targeting this problem aiming towards optimal bowel prep.

While sending an appointment date to the patient, information leaflets were also sent along with the bowel preparation, advising patients on the optimal method of using the agent for the most favourable results, avoiding certain foods and medications. This was designed in a simple, easy to understand manner, giving detailed and timed advice to patients starting almost a week before their procedure. Results showed an improved quality of bowel preparation as described later.

PDSA 2

We also noticed that money was spent on unnecessary packing while posting bowel preparation products to outpatients. These preparations were available by default in sachets quite safe for posting without using additional cardboard boxes worth $£ 5$ per postage. We hypothesised that our idea would help us save money without 
Figure 2 (Comparison of three measurements)

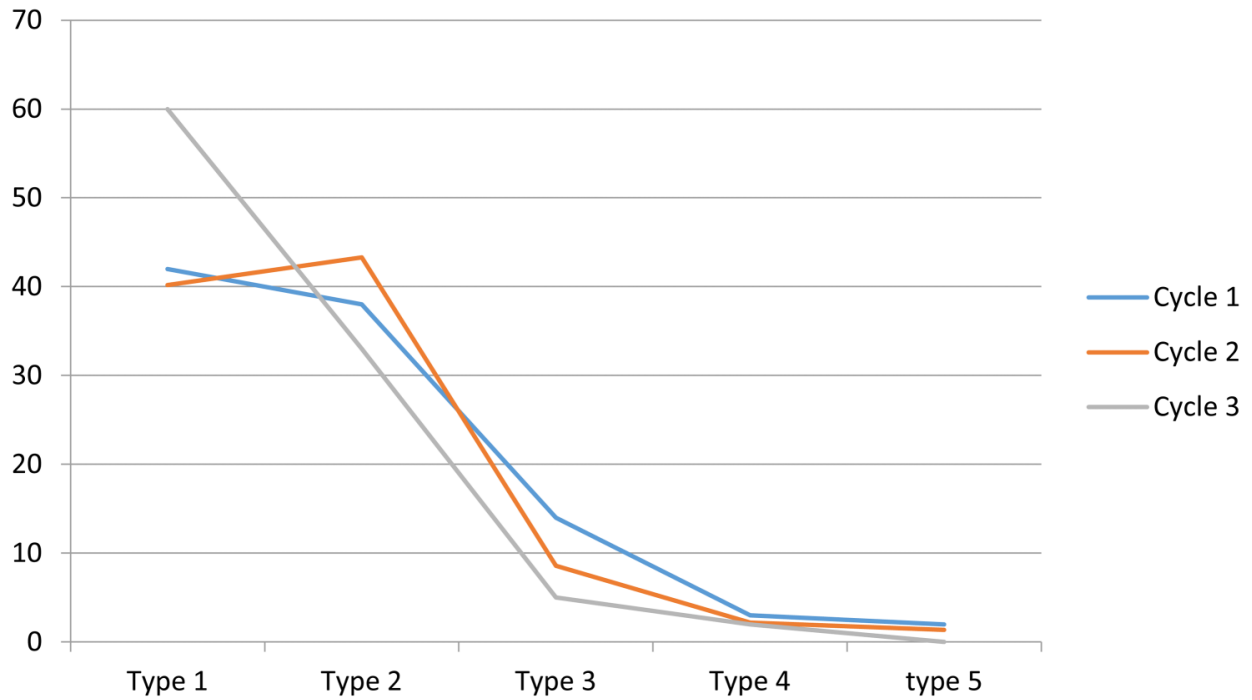

compromising the quality and safety of product delivery. With the help of our endoscopy management \& booking office we ran a pilot of these interventions and results as shown in next section were obviously promising.

\section{PDSA 3}

We also noticed that many times patients were prescribed preparations in a random manner depending upon the choice of requesting physician. We moved from a practice of individual triaging, to an algorithm based system, which provided more uniform prescriptions, avoiding unnecessary use of more expensive preparations where not indicated. This triaging was done by consultants in gastroenterology while authorising the procedure.

This cycle again focused at cost effectiveness of the procedure without affecting the quality of bowel preparation significantly as shown in next section (See supplementary file "Patient information leaflets").

\section{RESULTS}

In a repeat audit 3 months after the above interventions, we reviewed the records of 873 patients over the preceding 3 months and the results showed improving quality of bowel preparation. On this occasion $83.5 \%$ (729) patients had adequate bowel preparation while $11.60 \%$ (106) patients had inadequate bowel cleansing for optimal assessment. Only $20(2.29 \%)$ patients required repeat investigations.

We re-audited the same variables after 6 months at which point all interventions had been implemented. We looked into the records of the last 100 colonoscopies and the results on this occasion showed significant improvement from baseline measurements. 93(93\%) patients showed adequate bowl preparation while only $7 \%$ had inadequate bowel preparation.

None of the patients required a repeat investigation.
Though overall results were not significantly different in the first repeat measurement, there was a noticeable reduction in poor quality bowel preparation and results were much more encouraging in the 2nd phase. (Figure 2)

In terms of financial implications, we estimated that based on performing 4500 colonoscopies per year, changing the postage method alone would save $£ 23,265$ and changing prescription methods a further $£ 12,000$. (Figure 3)

The biggest saving, however would come from the reduction in number of repeat procedures required, saving an estimated $£ 150,000$ each year.

In addition to above we also noticed that end results of using different preparations i.e PEG vs Non PEG were comparable though there was a marked difference in their cost. Moviprep showed good bowel prep in 78.02\% vs poor prep results in $14.63 \%$ patients respectively while in $7.35 \%$ no records were available. Similarly, Kleenprep showed good results in $85.73 \%$ vs. poor results $9.98 \%$ patients with no records for $4.29 \%$ patients while Citramag senna showed $76.92 \%$ vs $20.5 \%$ respectively with no records for $3.58 \%$ patients. (Figure 4 )

We used PEG based preparation in 466/637 (73.15\%) \& 830/877(94\%) patients as shown in our first \& second study respectively however this was significantly improved after intervention as depicted in our final snapshot study

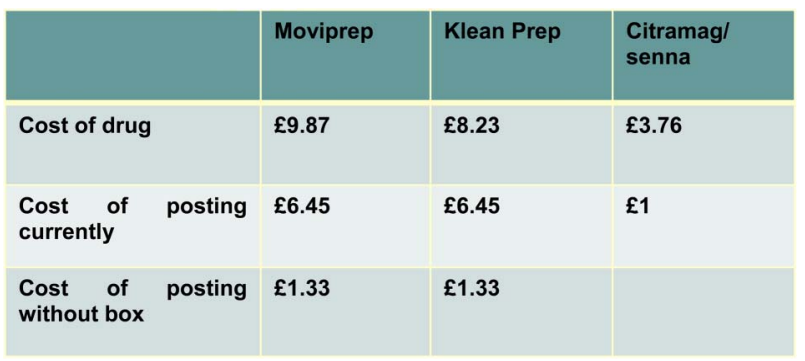

Figure 3 (Cost of the products and their postage) 


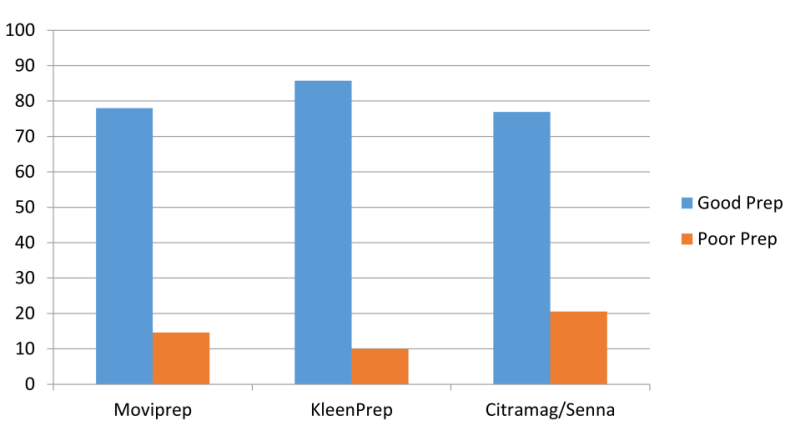

PDSA 2 Results: Total Patients 873

Type $1351(40.2 \%)$

Type $2378(43.3)$

Type $375(8.6 \%)$

Type $419(2.17 \%)$

Type $512(1.37 \%)$

\section{PDSA 3 Results: Total Patients 100}

$\begin{array}{ll}\text { Type } 1 & 60(60 \%) \\ \text { Type } 2 & 33(33 \%) \\ \text { Type 3 } & 5(5 \%) \\ \text { Type } 4 & 2(2 \%) \\ \text { Type } 5 & 0\end{array}$

Figure 4 (Comparing Moviprep, KleenPrep and Citramag/ Senna)

i.e $58.82 \%$. Though extremely cost effective with comparable efficacy, data is limited to prove that these results are valid on a wider scale and this needs further work.

\section{LESSONS AND LIMITATIONS}

Patient tolerance and compliance was an important factor in choosing bowel preparation and contributed towards poor results with patients not completing the regime.

In the last cycle we only looked at 100 patients in contrast to first 2 cycles and that can be a confounding factor in describing overall results.

Patient demographics (Age, Gender and Cognitive level) is a very important factor in compliance with prescribed preparation and was not looked at. Also this data includes some inpatient procedures. This might have affected the results based upon these variables however this process affected all cycles randomly.

Not everyone found it easy to comply with a specific diet plan a few days before the procedure.

This project can be sustained due to its attractive outcomes and minimal interventions required. The information leaflets have been incorporated on online portal system of the trust and with the help of endoscopy booking office are being distributed to patients alongside their appointments \& preparation material in a smooth manner.

This process is mainly applicable for elective outpatient settings and is suitable for hospitals where most of the outpatient lists are carried out. However, some principles of the project like "algorithm based prep selection" can still be applied for inpatients.
Though gastroenterology consultants have to authorise every endoscopy request, triaging every request tailored for individual patients was a time consuming process and we think this project can be expanded by giving simple guidance on the actual request form for physicians making a colonoscopy request.

Overall this project utilised very simple interventions and measurements with a focus on improving the quality of the procedure in a cost effective way.

The same process can be applied to other procedures including those related to gastroenterology and other specialities and generalisation of these interventions (for both quality and financial aspects) might prove extremely efficient for individual trusts as well as the NHS as a whole.

\section{CONCLUSION}

Poor bowel preparation is the leading cause of failed colonoscopies, resulting in delayed diagnosis and repeat investigations. A structured approach towards bowel preparation choice and patient education leads to better quality of bowel cleansing and is likely to improve the diagnostic yield of the procedures. This also means less numbers of repeat investigations also leading to a better financial outcome.

Appropriate choice of less expensive yet equally effective bowel cleaning preparations along with their delivery to the patients can also be optimised in a much better cost effective manner.

Acknowledgements Thanks to my fellow registrars Rachel Shakespeare and Hannah Glower in helping with the data collection alongside Endoscopy management and booking office in University Hospital Llandough.

Declaration of interests No Interests to declare.

Ethical approval Not Needed as no risks identified.

Open Access This is an open-access article distributed under the terms of the Creative Commons Attribution Non-commercial License, which permits use, distribution, and reproduction in any medium, provided the original work is properly cited, the use is non commercial and is otherwise in compliance with the license. See:

- http://creativecommons.org/licenses/by-nc/2.0/

- http://creativecommons.org/licenses/by-nc/2.0/legalcode

\section{REFERENCES}

1. Froehlich F, Wietlisbach V, Gonvers JJ . Impact of colonic cleansing on quality and diagnostic yield of colonoscopy: the European Panel of Appropriateness of Gastrointestinal Endoscopy European multicenter study. Gastrointest Endosc 2005; 61: 378-384.

2. Canard JM. Colonoscopy in France in 2008: results of the two-day survey of endoscopy in France. Acta Endosc 2010; 40: 58-65.

3. Lebwohl B, Kastrinos F, Glick M, Rosenbaum AJ, Wang T, Neugut Al. The impact of suboptimal bowel preparation on adenoma miss rates and the factors associated with early repeat colonoscopy. Gastrointest. Endosc. 73(6), 1207-1214 (2011).

4. Chokshi RV, Hovis CE, Hollander T, Early DS, Wang JS. Prevalence of missed adenomas in patients with inadequate bowel preparation on screening colonoscopy. Gastrointest. Endosc. 75(6), 1197-1203 (2012).

5. Rex DK, Imperiale TF, Latinovich DR . Impact of bowel preparation on efficiency and cost of colonoscopy. Am J Gastroenterol 2002; 97 : 1696-1700.

6. https://www.gov.uk/government/publications/bowel-cancer-screeningcolonoscopy-quality-assurance. (Last Accessed Feb 2011) 
7. British National Formulary, London: BMJ Publishing Group Ltd and Royal Pharmaceutical Society of Great Britain, 2009.

8. Consensus guidelines for the safe prescription and administration of oral bowel-cleansing agents, Connor A, Tolan D, Hughes S, Carr N, Tomson C, May 2012.

9. El Sayed AM, Kanafani ZA, Mourad FH, et al. A randomized single-blind trial of whole versus split-dose polyethylene glycol-electrolyte solution for colonoscopy preparation. Gastrointest Endosc 2003;58(1):36-40.

10. Vanner SJ, MacDonald PH, Paterson WG . A randomized prospective trial comparing oral sodium phosphate with standard polyethylene glycol-based lavage solution (Golytely) in the preparation of patients for colonoscopy. Am J Gastroenterol 1990;85:422-7.

11. Hsu CW, Imperiale TF. Meta-Analysis and cost comparison of polyethylene glycol lavage versus sodium phosphate for colonoscopy preparation. Gastrointest Endosc 1998;48: 276-82.

12. Edwin J.Lai MD, et al. The Boston Bowel Preparation Scale: $A$ valid and reliable instrument for colonoscopy-oriented research. Gastrointest Endosc. 2009 Mar; 69(3 Pt 2): 620-625. 